\title{
RIQUEZA DE ESPÉCIES DE OLIGOCHAETA (ANNELIDA, CLITEllatA) EM DUAS REPRESAS DO RIO TIETÊ (SÃO PAULO).
}

\author{
Paulo Augusto Zaitune Pamplin ${ }^{+}$Odete Rocha $a^{\ddagger}$ Mercedes Marches $\boldsymbol{e}^{\dagger}$
}

Biota Neotropica v5 (n1) - http://www.biotaneotropica.org.br/v5n1/pt/abstract?article+BN00605012005

Recebido: 09/09/2004

Revisado: 11/11/2004

Publicado: 01/01/2005

\begin{abstract}
* Universidade Federal de São Carlos, Departamento de Ecologia e Biologia Evolutiva, Via Washington Luís km 235, 13565-905, São Carlos, SP, Brasil. (e-mail: pazpamplin@click21.com.br; doro@power.ufscar.br)

† Instituto Nacional de Limnología (INALI-CONICET-UNL), José Maciá 1933, (3016) Santo Tomé (Santa Fe), Argentina
\end{abstract}

\begin{abstract}
The species richness of Oligochaeta was evaluated in two reservoirs belonging to Tietê River system, Bariri and Ponte Nova Reservoirs. 360 sediment samples were taken in each reservoir at 90 sampling stations using a Van Veen grab $\left(378 \mathrm{~cm}^{2}\right)$, in the months January, April, July and October 2001. A total of 12.826 oligochaete specimens were collected, belonging to 20 species, 10 genera and 4 families. The family and genus with the highest species richness were Naididae and Dero, with 14 and 9 species, respectively. Among all species identified 6 occurred in both reservoirs, being Branchiura sowerbyi (Tubificidae) the most abundant species in them. The study also revealed that most Oligochaeta species are rare and have accidental occurrence, thus evidencing that for a better knowledge of species richness is actually necessary a great sampling effort including a large number of sampling points.
\end{abstract}

\section{Resumo}

A riqueza de espécies de Oligochaeta foi investigada em duas represas construídas no curso do rio Tietê. Em cada represa foram obtidas 360 amostras de sedimento em 90 pontos de amostragem, utilizando-se uma draga Van Veen $\left(378 \mathrm{~cm}^{2}\right)$, nos meses de janeiro, abril, julho e outubro de 2001. No total foram examinados 12.826 exemplares de oligoquetos pertencentes a 20 espécies, 10 gêneros e 4 famílias. Naididae e Dero foram a família e o gênero com maiores riquezas de espécies, com 14 e 9 espécies, respectivamente. Do total de espécies identificadas, 6 estiveram presentes em ambas represas, sendo Branchiura sowerbyi (Tubificidae) a espécie mais abundante nestes ambientes. A análise das amostras revelou que a maioria das espécies de oligoquetos é rara e de freqüência de ocorrência acidental, evidenciando que para um melhor conhecimento da riqueza de espécies é realmente necessário um grande esforço amostral, com elevado número de pontos de amostragem. 


\section{Introdução}

A Classe Oligochaeta, juntamente com as larvas de Chironomidae, constituem os principais componentes da fauna de invertebrados em diferentes tipos de habitats, fazendo parte de comunidades como o bentos, o perifiton e o pleuston, tanto em ambientes lênticos como lóticos (Harman 1982). Estes organismos também podem ser registrados em limnótopos originados pelas bromeliáceas e entre as folhas e troncos de árvores em decomposição. Em relação ao hábito alimentar, a maioria das espécies é detritívora, mas alguns Naididae se alimentam de algas, enquanto outros são comensais e predadores (Gavrilov 1977, Marchese 1995).

De acordo com Righi (2002), no Brasil são atualmente conhecidas cerca de 70 espécies de oligoquetos, das quais 46 têm ocorrência registrada no estado de São Paulo. Os primeiros registros de Oligochaeta em ambientes aquáticos brasileiros datam do início do século XX, mas somente entre as décadas de 40 e 60 é que houve uma maior ampliação do conhecimento da fauna de oligoquetos limnícos no Brasil, devido aos trabalhos realizados pelos pesquisadores Marcus e du Bois-Reymond Marcus (Righi, 2002). Mais recentemente, entre as décadas de 70 e 90 , os estudos realizados pelo professor Gilberto Righi consolidaram o atual conhecimento da taxonomia e sistemática de Oligochaeta no Brasil (Moreno \& Mischis 2004, Römbke 2004). Desde então, alguns trabalhos sobre a composição, distribuição e ecologia dos Oligochaeta tem sido realizados, como os de Takeda (1999), Alves \& Strixino (2000) Montanholi-Martins \& Takeda (2001); Collado \& Schmelz (2001), Corbi \& Trivinho-Strixino (2002).

O presente trabalho tem como objetivo avaliar a riqueza de espécies de Oligochaeta em dois reservatórios da Bacia Hidrográfica do rio Tietê.

\section{Materiais e Métodos}

Os exemplares de Oligochaeta foram coletados em

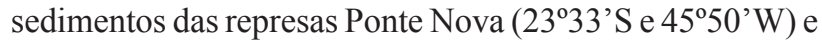
Bariri (22 $06^{\circ} \mathrm{S}$ e $\left.48^{\circ} 45^{\prime} \mathrm{W}\right)$ nos meses de janeiro, abril, julho e outubro de 2001. Ambas as represas foram construídas no curso do rio Tietê entre o final da década de 60 e o início da década de 70. As principais características destas represas estão sumarizadas na tabela I.

As amostras de sedimento, no total 360, foram coletadas em 90 pontos de amostragens em cada represa (Figura 1) com uma draga Van Veen $\left(378 \mathrm{~cm}^{2}\right)$. Na represa Ponte Nova as amostras foram obtidas entre as latitudes

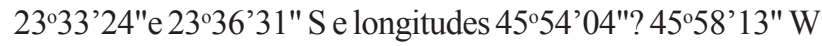
e na represa Bariri entre as coordenadas $22^{\circ} 09^{\prime} 19^{\prime \prime}$ ?

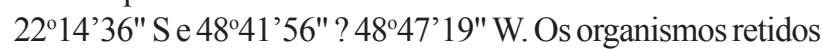
em peneira com $210 \mathrm{~mm}$ de abertura de malha foram preservados em formalina $10 \%$ e, posteriormente, separados, identificados e contados. Exemplares dos Oligochaeta foram montados em lâminas semi-permanentes em solução Hoyer (Trivinho-Strixino \& Strixino 1995) para análise dos caracteres morfológicos de importância taxonômica para identificação (Righi 1984, Brinkhurst \& Marchese 1992). A abundância relativa e a freqüência de ocorrência das espécies foram calculadas e categorizadas de acordo com McCullough \& Jackson (1985) e Jablonska \& Paturej (1999).

\section{Resultados e Discussão}

No total, 12.826 exemplares de oligoquetos foram examinados, sendo 12.138 coletados na represa Bariri e 688 na represa Ponte Nova. A maioria dos indivíduos se encontravam inteiros e sexualmente maduros, tendo sido identificadas 20 espécies pertencentes a 10 gêneros e 4 famílias (Tabela II). A família Naididae foi aquela com maior número de espécies, 12 na represa Bariri e 4 na represa Ponte Nova, enquanto que Tubificidae esteve representada por 3 e 4 espécies, respectivamente. Em ambas represas, Opistocystidae foi representada apenas por Opistocysta funiculus e na represa Ponte Nova foi registrada a ocorrência de uma espécie de megadrilideo, Brinkhurstia americanus (Alluroididae).

Entre as espécies identificadas, 6 espécies estiveram presentes em ambas as represas, sendo que a ocorrência de Allonais lairdi representa o primeiro registro desta espécie para o Brasil e as ocorrências de Dero (Dero) pectinata e D. (D.) digitata representam o primeiro registro para o Estado de São Paulo.

\subsection{Considerações sobre as espécies}

Naididae (= Naidinae de acordo com Erséus \& Källersjö 2004, Erséus \& Gustavsson 2002)

Allonais lairdi Naidu, 1965. Indivíduos desta espécie foram encontrados somente na represa Ponte Nova, sendo o primeiro registro desta espécie para o Brasil. A. lairdi tinha sido anteriormente registrada na América do Sul somente na Argentina (Brinkhurst \& Marchese 1992). Armendariz (1999) estudando a dinâmica populacional desta espécie em um pequeno ambiente lêntico, próximo a Buenos Aires (Argentina), observou a existência de 3 coortes anuais com um curto período de maturidade sexual.

Allonais paraguayensis (Michaelsen, 1905). Espécie amplamente distribuída com registros na América do Sul e do Norte, Austrália, África e China (Righi 1984, Brinkhurst \& Marchese 1992, Milligan 1997, Timm 1999). Neste estudo apenas 6 indivíduos de $A$. paraguayensis foram coletados na represa Bariri.

Dero (Aulophorus) furcatus Müller, 1773. Espécie cosmopolita. No presente trabalho ocorreu somente na represa Bariri em número reduzido, com 6 indivíduos. Além do estado de São Paulo, Righi (1984) aponta o registro desta espécie também para os estados de Pernambuco, Pará e Amazonas. 


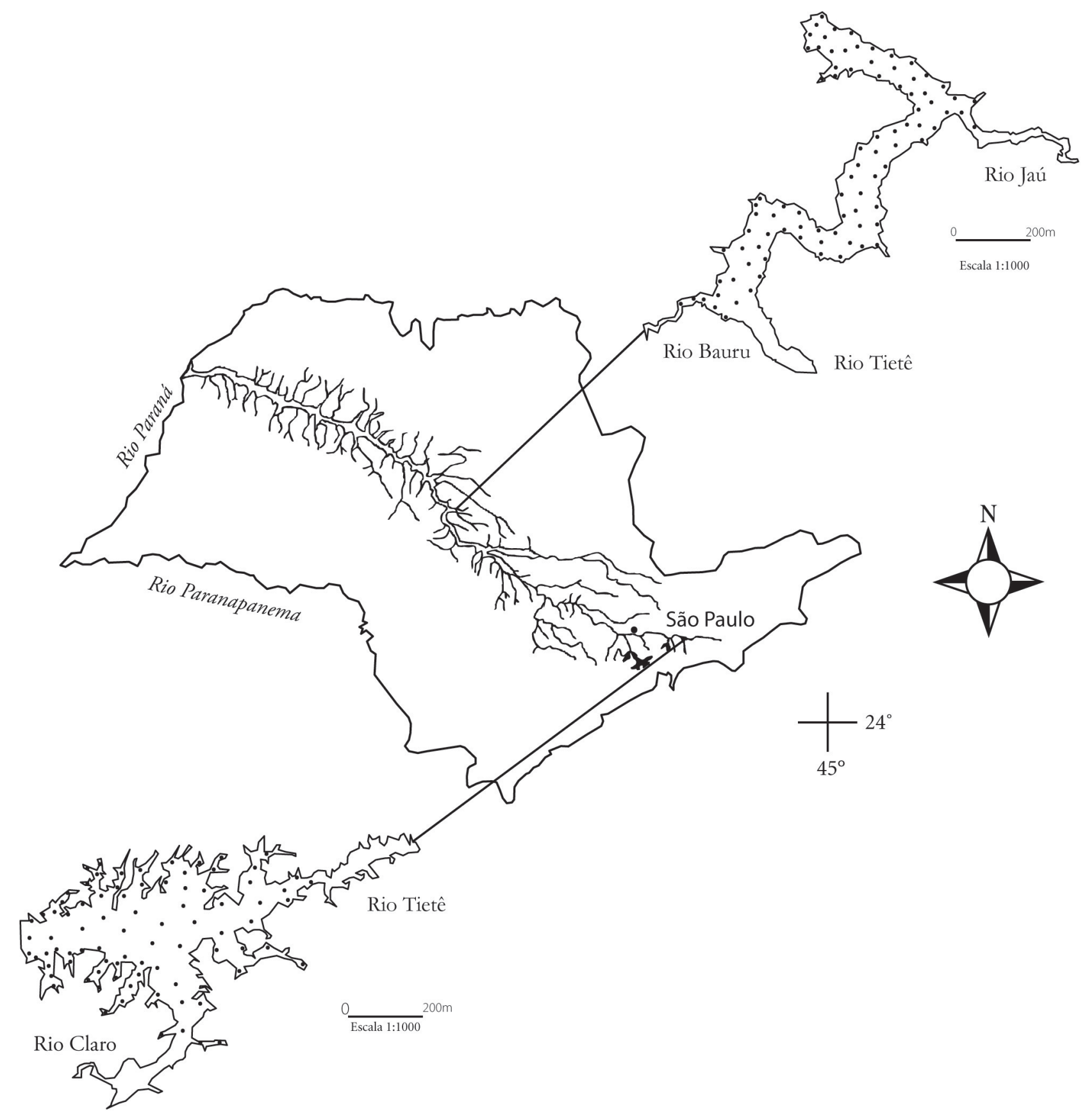

Figura 1 - Mapa das represas Ponte Nova e Bariri com a localização dos pontos de coleta. 


\begin{tabular}{lcc}
\hline & Represa Ponte Nova & Represa Bariri \\
\cline { 2 - 3 } Ano de conclusão & 1972 & 1969 \\
Altitude $(\mathrm{m})$ & 765 & 420 \\
Área total $\left(\mathrm{Km}^{2}\right)$ & 25,7 & 62,5 \\
Área de drenagem $\left(\mathrm{Km}^{2}\right)$ & 320 & 35.430 \\
Vazão média anual $\left(\mathrm{m}^{3} . \mathrm{s}^{-1}\right)$ & 3,4 & 327,0 \\
Tempo de residência $($ dias $)$ & - & 7 a 24 \\
Volume total $\left(\mathrm{x} 10^{6} \mathrm{~m}^{3}\right)$ & 336,4 & 542,0 \\
Perímetro $(\mathrm{Km})$ & - & 193 \\
Profundidade média $(\mathrm{m})$ & 13,1 & 8,6 \\
Principais tributários & Rio Claro & Rios Lençóis, Jaú, Bauru e \\
& & Ribeirão Grande \\
\hline
\end{tabular}

Dero (Aulophorus) lodeni Brinkhurst, 1986. Esta espécie tem sido registrada na América do Sul e do Norte, além da África (Milligan 1997, Brinkhurst \& Marchese 1992). Righi (1984) menciona a ocorrência desta espécie no Brasil, pelo uso da sinonimia $D$. (A.) pectinatus. No presente estudo, esta espécie foi somente registrada na represa Bariri, ocorrendo em $16,67 \%$ da amostras, sendo entre as espécies de Naididae encontradas nesta represa, aquela para a qual se obteve a maior freqüência de ocorrência.

Dero (Dero) botrytis Marcus, 1943. Espécie descrita em material coletado no Brasil. Também tem sido registrada em ambientes aquáticos na Argentina e no Uruguai (Righi 1984, Brinkhurst \& Marchese 1992). Milligan (1997) observou a presença $D$. (D.) botrytis em baixas densidades, em rios e córregos com substrato arenoso fino e limpo, na região central da Florida. Esta espécie se distingue das demais espécies de Dero (Dero) pelo grande número de brânquias, usualmente cerca de 40 pares (Brinkhurst \& Marchese 1992).

Dero (Dero) digitata (Muller, 1773). Espécie cosmopolita. Milligan (1997) cita que na Florida ocorrem em densas populações. Na represa Bariri foram registrados somente 20 indivíduos $(0,16 \%)$ em apenas 2 amostras $(0,56 \%)$, sendo este o primeiro registro desta espécie para o estado de São Paulo. Righi (1984) cita a presença desta espécie em rios e lagos no Amazonas e Pará. Takeda (1999) registrou-a na porção superior do rio Paraná.

Dero (Dero) evelinae Marcus, 1943. 11 exemplares desta espécie foram encontrados na represa Bariri. De acordo com Righi (1984), esta espécie tem ampla distribuição no Brasil, com registros nos estados do Pará, Piauí, Alagoas, Pernambuco, São Paulo, Paraná e Rio Grande do Sul, além da Argentina e Uruguai.
Dero (Dero) multibranchiata Steiren, 1982. Espécie restrita à América do Sul (Brinkhurst \& Marchese 1992). Neste estudo esteve presente apenas na represa Ponte Nova, com baixa densidade. Corbi \& Trivinho-Strixino (2002) também registram esta espécie no Estado de São Paulo. Anteriormente a estas ocorrências, no Brasil D. (D.) multibranchiata apenas havia sido registrada nos estados de Pernambuco, Pará, Amazonas e Roraima (Righi 1984).

Dero (Dero) nivea Aiyer, 1929. Espécie cosmopolita, geralmente associada às regiões marginais de sistemas aquáticos com macrófitas e sedimento fino (Milligan 1997). Brinkhurst \& Marchese (1992) mencionam a ocorrência desta espécie apenas na Argentina e Suriname. No Brasil esta espécie foi anteriormente registrada em baixas densidades, na lagoa do Diogo (Alves \& Strixino 2000) e na represa do ribeirão Anhumas (Corbi \& Trivinho-Strixino 2002), ambas no Estado de São Paulo. Na represa Bariri, esta espécie representou aproximadamente $1,5 \%$ dos Oligochaeta coletados e ocorreu em menos de $1,8 \%$ das amostras.

Dero (Dero) obtusa d'Udekem, 1885. Espécie cosmopolita, ocorreu na represa Bariri em apenas 4 amostras (1,11\%). Na América do Sul e Central, Brinkhurst \& Marchese (1992) apontam a presença desta espécie na Argentina, Brasil, Bolívia, Costa Rica, Haiti, Suriname e Venezuela. No Brasil, esta espécie tem sido registrada apenas para o estado São Paulo (Righi 1984, Corbi \& Trivinho-Strixino 2002).

Dero (Dero) pectinata Aiyer, 1929. No Brasil, esta espécie havia sido registrada somente na lagoa dos Patos, na Bacia do rio Paraná (Montanholi-Martins \& Takeda 2001). Sete indivíduos foram coletados na represa Bariri, constituindo o primeiro registro para o estado de São Paulo. 
Tabela 2 - Abundância relativa e freqüência de ocorrência das espécies de Oligochaeta nas represas Bariri e Ponte Nova em 2001. Escala de Abundância Relativa: Dominantes(T)(> 50\%), Abundantes (V)(49,99-30\%), Comuns (TM)(29,99-10\%), Ocasionais (£)(9,99-1\%) e Raros ()$(<1 \%$ ) (adaptado de McCullough \& Jackson, 1985). Escala de Freqüencia de Ocorrência: Euconstantes $(\phi)(>61 \%)$, Constantes ( ) (60,99-41\%) Acessórias (¿)(40,99-21\%) e Acidentais (U்)(> 20,99\%) (adaptado de Jablonska \& Paturej 1999).

\begin{tabular}{|c|c|c|c|c|c|c|c|c|}
\hline & \multicolumn{4}{|c|}{ Abundância Relativa(\%) } & \multicolumn{4}{|c|}{ Freq. Ocorrência (\%) } \\
\hline & \multicolumn{2}{|c|}{ Bariri } & \multicolumn{2}{|c|}{ Ponte Nova } & \multicolumn{2}{|c|}{ Bariri } & \multicolumn{2}{|c|}{ Ponte Nova } \\
\hline \multicolumn{9}{|l|}{ Naididae } \\
\hline Allonais lairdi & & & 1,31 & $\square$ & & & 1,11 & $\star$ \\
\hline Allonais paraguayensis & 0,05 & $\diamond$ & & & 0,56 & $\star$ & & \\
\hline Dero (Aulophorus) furcatus & $\mathbf{0 , 0 5}$ & $\diamond$ & & & 0,56 & $\star$ & & \\
\hline Dero (Aulophorus) lodeni & 1,12 & $\square$ & & & 16,67 & $\star$ & & \\
\hline Dero (Dero) botrytis & 0,48 & $\diamond$ & & & 2,78 & * & & \\
\hline Dero (Dero) digitata & 0,16 & $\diamond$ & & & 0,56 & * & & \\
\hline Dero (Dero) evelinae & 0,09 & $\diamond$ & & & $\mathbf{0 , 8 3}$ & * & & \\
\hline Dero (Dero) multibranchiata & & & 0,29 & $\diamond$ & & & 0,56 & $\star$ \\
\hline Dero (Dero) nívea & 1,42 & $\square$ & & & 1,67 & * & & \\
\hline Dero (Dero) obtusa & 0,27 & $\diamond$ & & & 1,11 & * & & \\
\hline Dero (Dero) pectinata & 0,06 & $\diamond$ & & & 0,56 & * & & \\
\hline Haemonais waldvogeli & 0,47 & $\diamond$ & & & 1,67 & * & & \\
\hline Prístina americana & 4,16 & $\square$ & 0,15 & $\diamond$ & 5,83 & * & $\mathbf{0 , 2 8}$ & * \\
\hline Slavina evelinae & 0,05 & $\diamond$ & 0,29 & $\diamond$ & $\mathbf{0 , 8 3}$ & * & $\mathbf{0 , 5 6}$ & $\star$ \\
\hline \multicolumn{9}{|l|}{ Tubificidae } \\
\hline Bothrioneurum americanum & 0,31 & $\diamond$ & 3,20 & $\square$ & 0,56 & * & 2,23 & $\star$ \\
\hline Branchiura sowerbyi & 72,34 & 凶 & 53,63 & 凶 & 70,28 & $\mathbf{\square}$ & 40,39 & $\diamond$ \\
\hline Limnodrilus hoffmeisteri & 18,36 & $\bigcirc$ & 37,06 & $\otimes$ & 55,56 & ○ & 18,38 & * \\
\hline Limnodrilus udekemianus & & & 0,29 & $\diamond$ & & & 0,56 & * \\
\hline \multicolumn{9}{|l|}{ Opistocystidae } \\
\hline Opistocysta funiculus & 0,60 & $\diamond$ & 1,02 & $\square$ & 5,00 & * & 1,11 & * \\
\hline \multicolumn{9}{|l|}{ Alluroididae } \\
\hline Brinkhurstia americanus & & & 2,76 & $\square$ & & & 1,67 & * \\
\hline
\end{tabular}


Haemonais waldvogeli Bretscher, 1900. Espécie com ampla distribuição mundial (Milligan 1997). Recentemente, teve o primeiro registro no Japão (Akifumi \& Machiko 1999) e na Austrália (Pinder 2003). No Brasil, esta espécie havia sido anteriormente registrada por Montanholi-Martins \& Takeda (2001) para a lagoa dos Patos, na Bacia hidrográfica do Rio Paraná. Na represa Bariri foram encontrados 57 exemplares desta espécie, a qual não ocorreu na represa Ponte Nova.

Pristina americana Cernosvitov, 1937. Righi (1984) registra a ocorrência de 11 espécies do gênero Pristina para o Brasil. Collado \& Schmelz (2001) reportaram em amostras de solo e serrapilheira da Floresta Amazônica a presença de P.jenkinae, P. notopora e P. marcusi, esta última, uma espécie nova. Na represa Ponte Nova foi encontrado apenas 1 indivíduo de Pristina americana, enquanto que na represa Bariri P. americana foi a espécie mais abundante de Naididae, tendo sido coletados 505 indivíduos, distribuídos em apenas $5,83 \%$ das amostras, representando $4,16 \%$ do total.

Slavina evelinae (Marcus, 1942). Espécie registrada na Argentina, Suriname, Antigua e Brasil (Brinkhurst \& Marchese 1992, Takeda 1999, Corbi \& Trivinho-Strixino 2002). Na represa Bariri foram coletados 6 indivíduos e na represa Ponte Nova 2 indivíduos, correspondendo a menos de $1 \%$ do total de Oligochaeta coletados em ambas represas.

\section{TUBIFICIDAE}

Bothrioneurum americanum Beddard, 1894. As espécies do gênero Bothrioneurum são facilmente reconhecidas pela presença de uma pequena depressão (órgão sensitivo) localizada na região médio-dorsal e pelos espermatóforos externos (Brinkhurst \& Marchese 1992). Estes autores enfatizam que este gênero necessita de uma revisão taxonômica. No presente estudo, B. americanum ocorreu em ambas as represas, em baixa densidade.

Branchiura sowerbyi Beddard, 1892. Espécie cosmopolita, facilmente reconhecida pela presença de brânquias dorsais e ventrais nos segmentos posteriores. Esta espécie foi o principal componente da Classe Oligochaeta em ambas as represas, com abundância relativa igual a 53,6\% na represa Ponte Nova e 72,3\% na represa Bariri. Em ambas as represas, foi a espécie mais freqüente, ocorrendo em 70\% das amostras da represa Bariri e em 40\% na represa Ponte Nova. Os registros desta espécie no Brasil restringem-se ao Estado de São Paulo (Righi 1984).

Limnodrilus hoffmeisteri Claparede, 1862. Espécie cosmopolita. De acordo com Wetzel \& Taylor (2001), esta espécie é provavelmente a mais comumente encontrada em ambientes aquáticos, dominando em ambientes organicamente enriquecidos (Milbrink 1994, Marchese \& Ezcurra de Drago 1999, Brinkhurst \& Marchese 1992, Milbrink et al. 2002). No Brasil existem registros em diferentes localidades (Righi 1984). Esta foi a segunda espécie mais abundante e freqüente nas represas Ponte Nova e Bariri.
Limnodrilus udekemianus Claparede, 1862. Espécie cosmopolita, ocorrendo em diferentes tipos de ambientes, mas raramente abundante (Milligan 1997, Wetzel \& Taylor 2001). Pode ser diferenciada de L. hoffmeisteri pelo tamanho reduzido da bainha peniana em indivíduos sexualmente maturos. No material analisado apenas dois indivíduos sexualmente maduros de L. udekemianus foram coletados na represa Ponte Nova. No Brasil, até o momento, esta espécie foi registrada apenas no estado de São Paulo (Righi 1984).

\section{OPISTOCYSTIDAE}

Opistocysta funiculus Cordero, 1948. Brinkhurst \& Marchese (1992) informam que o gênero Opistocysta tem distribuição restrita ao continente sul-americano, com registro no Paraguai (O. serrata Harman, 1969), na Argentina e Brasil (O. funiculus Cordero, 1948). Tanto na represa Ponte Nova como na represa Bariri, a ocorrência desta espécie foi rara, com registro de 7 e 73 indivíduos, respectivamente.

\section{Alluroididae}

Brinkhurstia americanus (Brinkhurst 1964). Espécie encontrada na Argentina, Guiana e Brasil (Righi 1984, Brinkhurst \& Marchese 1992) e no Panamá (Rodriguez 2002). No presente estudo sua ocorrência foi registrada apenas na represa Ponte Nova, sendo que os 19 indivíduos analisados eram sexualmente imaturos.

\section{Conclusões}

Nas represas estudadas a ocorrência de maior número de espécies pertencentes à família Naididae corrobora o padrão freqüentemente reportado na literatura científica, que indica ser esta a principal família componente dos oligoquetos em relação à riqueza de espécies (e.g., Marchese 1995, Timm et al. 2001, Peralta et al. 2002, Spencer \& Hudson 2003, Nijboer et al. 2004). O presente estudo evidenciou que a maior parte das espécies encontradas são raras e com freqüência de ocorrência acidental, o que indica que para um melhor conhecimento da riqueza de espécies é necessário um maior esforço amostral (maior número de estações de coleta).

\section{Agradecimentos}

Os autores agradecem à FAPESP (Proc No 99/123938) pela concessão de bolsa de estudo para o primeiro autor e suporte financeiro. Também agradecem ao Prof. Dr. Vangil Pinto da Silva (in memoriam), da Universidade Federal do Mato Grosso, pela infra-estrutura cedida quando da identificação do matéria e ao MMA/CNPq pelo suporte financeiro por meio dos programas PRONEX e PROBIO para os estudos de biodiversidade. 


\section{Referências Bibliográficas}

AKIFUMI, O.\& MACHIKO, N. 1999. Studies on the aquatic oligochaetes fauna in Lake Biwa, central Japan. II. Records and taxonomic remarks of nine species. Hydrobiologia, 406:33-47.

ALVES, R.G. \& STRIXINO, G. 2000. Distribuição espacial em uma lagoa marginal do Rio Mogi-Guaçu. Iheringia (Série Zoologia), 88:173-180

ARMENDARIZ, L.C. 1999. Population dynamics of Allonais lairdi (Oligochaeta, Naididae) from Los Talas, Buenos Aires Province. Ecologia Austral 9(1-2): 20-27.

BRINKHURST, R.O. \& MARCHESE, M. 1992. Guía para la identificación de oligoquetos acuáticos continentales de Sud y Centroamérica. 2. ed. Asociacíon de Ciências Naturales del Litoral, Colección Climax 6.

COLLADO, R. \& SCHMELZ, R.M. 2001. Descriptions of three Pristina species (Naididae, Clitellata) from Amazonian forest soils, including $P$. marcusi sp. nov. Hydrobiologia 463:1-11.

CORBI, J.J. \& TRIVINHO-STRIXINO, S. 2002. Spatial and bathymetric distribution of the macrobenthic fauna of the Ribeirão das Anhumas Reservoir (Américo Brasiliense - SP, Brazil). Acta Limnol. Bras. 14(1):35-42.

ERSÉUS, C.\& GUSTAVSSON, L. 2002. A proposal to regard the former family Naididae as a subfamily within Tubi.cidae (Annelida, Clitellata). Hydrobiologia, 485:253-256.

ERSÉUS, C. \& KÄLLERJÖ, M. 2004. 18S rDNA phylogeny ofClitellata (Annelida). Zoologica Scripta, 33 (2):187-196.

GAVRILOV, K. 1977. Oligochaeta. In Biota Acuática de Sudamérica Austral (S.H. HURLBERT, ed.). San Diego State University, p.99-121.

HARMAN, W. 1982. Oligochaeta. In Aquatic of México, Central America and the West Indies (S.H. HURLBERT \& A. VILLALOBOS-FIGUEROS, eds.). San Diego State University, p.162-165.

JABLONSKA, I. \& PATUREJ, E. 1999. The domination and constancy of occurrence of invertebrate communities in the Hancznska Bay, Lake Wigry, NW Poland. Acta Hydrobiol., 41(6):249-253.

MARCHESE, M. 1995. Annelida Oligochaeta. In Ecosistemas de aguas continentales. Metodologías para su estudio (E. LOPRETTO \& G. TELL, Directores). Ediciones Sur, La Plata, tomo II, p.709-731.

MARCHESE, M. \& EZCURRA DE DRAGO, I. 1999. Use of benthic macroinvertebrates as organic pollution indicators in lotic environments of the Parana River drainage basin. Polskie Archiwum Hydrobiologii 46(3-4):233-255.

MCCULLOUGH, J.D. \& JACKSON, D.W. 1985. Composition and producticity of the benthic macroinvertebrate community of subtropical reservoir. Int. Revue ges. Hydrobiol. 70(2):221-235.
MILBRINK, G. 1994. Oligochaetes and water pollution in two deep Norwegian lakes. Hydrobiologia 278:213-222.

MILBRINK, G., TIMM, T., \& LUNDBERG, S. 2002. Indicative profundal oligochaete assemblages in selected small Swedish lakes. Hydrobyologia 468:53-61.

MILLIGAN, M.R. 1997. Identification manual for the aquatic Oligochaeta of Florida. Volume 1. Freshwater oligochaetes. Florida Department Environmental. Protection, USA.

MONTANHOLI-MARTINS, M.C. \& TAKEDA, A. M. 2001. Spatial and temporal variations of oligochaetes of the Ivinhema River and Patos Lake in the Upper Parana River Basin, Brazil. Hydrobiologia 463:197-205.

MORENO,A.G. \& MISCHIS, C.C. 2004. The status of Gilberto Righi's collection at the museum of São Paulo. Righi memorial: tropical ecology. Pedobiologia 47(5-6):413-418.

NIJBOER, R.C., WETZEL, M.J. \& VERDONSCHOT, P.F.M. 2004. Diversity and distribution of Tubificidae, Naididae, and Lumbriculidae (Annelida: Oligochaeta) in the Netherlands: an evaluation of twenty years of monitoring data. Hydrobiologia 520:127-141.

PERALTA, L., ESCOBAR, E.,ALCOCER, J. \& LUGO, A. 2002. Oligochaetes from six tropical crater lakes in Central Mexico: species composition, density and biomass. Hydrobiologia 467:109-116.

PINDER, A.M. 2003. First Australian records of three species and two genera of aquatic oligochaetes (Clitellata: Annelida). Proceedings of the Linnean Society of New South Wales 124:109-114.

RIGHI, G. 1984. Oligochaeta. In Manual de identificação de invertebrados límnicos do Brasil, 17 (In R. Schaden, ed.). CNPq, Brasília.

RIGHI, G. 2002. Anelídeos Oligoquetos. In Biodiversidade do Estado de São Paulo, Brasil. Invertebrados de água doce. v. 4 (D. Ismael, W. C. Valenti, T Matsumura-Tudisi, O. Rocha, eds.). FAPESP, São Paulo.

RODRIGUEZ, P. 2002. Benthic and subterranean aquatic oligochaete fauna (Annelida, Oligochaeta) from Coiba island (Panama) and Cuba. Graellsia 58(2):3-19.

RÖMBKE, J. 2004. The role of Gilberto Righi in the development of tropical taxonomy. Pedobiologia 47(5-6):405-412.

SPENCER, D.R. \& HUDSON, P.L. 2003. The Oligochaeta (Annelida, Clitellata) of the St. Lawrence Great Lakes region: an update. J. Great Lakes Res. 29(1):89-104.

TAKEDA, A. M. 1999. Oligochaete community of alluvial Upper Paraná River, Brazil: spatial and temporal distribution (1987-1988). Hydrobiologia 412: 35-42..

TIMM, T. 1999. Distribution of freshwater oligochaetes in the west and east coastal regions of the North Pacific Ocean. Hydrobiologia 406:67-81. 
TIMM, T., SEIRE, A. \& PALL, P. 2001. Half a century of oligochaete research in Estonian running waters. In Aquatic Oligochaete Biology VIII (P. RODRIGUEZ \& P.F.M. VERDONSCHOT, eds.). Kluwer Academic Publisher, Dordrecht, Hydrobiologia 463:223-234.

TRIVINHO-STRIXINO, S. \& STRIXINO, G. 1995. Larvas de Chironomidae (Diptera) do Estado de São Paulo: guia para identificação e diagnose dos gêneros. PPG-ERN/ UFSCAR, São Carlos.

WETZEL, M.J. \&TAYLOR, S.J. 2001. First records of freshwater oligochaetes (Annelida, Clitellata) from caves in Illinois and Missouri, USA. Journal of Cave and Karst Studies 63(3):99-104.

Título: Riqueza de espécies de Oligochaeta (Annelida, Clitellata) em duas represas do rio Tietê (São Paulo).

Autores: Paulo Augusto Zaitune Pamplin, Odete Rocha e Mercedes Marchese

Biota Neotropica, Vol. 5 ( número1): 2005

http://www.biotaneotropica.org.br/v5n 1/pt/ abstract?article+BN00605012005

Recebido: 09/09/2004

Revisado: 11/11/2004

Publicado: 01/01/2005

ISSN 1676-0603 